Mongolian Geoscientist

Original article

\title{
Age, origin and tectonic setting of Dulaankhan granitic pluton in northern Mongolia
}

\author{
Baatar Gendenjamts $^{1 *}$, Baatar Munkhtsengel ${ }^{2}$, Dashdorjgochoo Odgerel ${ }^{1}$, \\ Dorjgochoo Sanchir ${ }^{1}$, Bayaraa Ganbat ${ }^{3}$
}

\author{
${ }^{1}$ Department of Magmatism and Metallogeny, Institute of Geology, Mongolian Academy of Sciences, Ulaanbaatar 15160, Mongolia \\ ${ }^{2}$ Department of Geology and Hydrogeology, School of Geology and Mining Engineering, Mongolian University of Science and Technology \\ Ulaanbaatar 14191, Mongolia \\ ${ }^{3}$ Faculty of Geochemistry and Environment, School of Earth and Space Sciences, University of Science and Technology of China, Hefei 230026, \\ China \\ *Corresponding author. Email: gendenjamtsb@mas.ac.mn
}

\section{ARTICLE INFO}

Article history:

Received 10 September 2019

Accepted 11 October 2019

\begin{abstract}
Dulaankhan granitic pluton, which is situated in northern Mongolia, the southern portion of the Mongolian-Transbaikalian belt (MTB), is petrographically composed of fine to medium-grained peralkaline granite and is intruded by a small body of quartz syenite. Geochemical data show the Dulaankhan granite and the intruding quartz syenite are both slightly peraluminous and high-K calc-alkaline, and are enriched in LREEs relative to the HREEs, with negative Eu anomaly, and in large ion lithophile elements (LILEs; such as $\mathrm{K}$, Cs and $\mathrm{Rb}$ ) with respect to high field strength elements (HFSEs; e.g., $\mathrm{Nb}$, Ta and Ti). In terms of relations of $\mathrm{Nb}, \mathrm{Zr}$ and $\mathrm{Y}$ to $\mathrm{Ga} / \mathrm{Al}$, however, the Dulaankhan granite and quartz syenite show geochemical features of A-type granites and can be classified into the A2-sub type granite, implying that the pluton formed in an post-collision extensional environment. LAICPMS zircon $\mathrm{U}-\mathrm{Pb}$ dating results suggest that the Dulaankhan granite crystallized at $198 \pm 1 \mathrm{Ma}$, whereas the intruding quartz syenite at $180 \pm 1 \mathrm{Ma}$, consistent with our field observation that the quartz syenite intrudes the granite, attesting that the two granitic bodies were emplaced at different times although both of them formed during the Early Jurassic period. According to these new data, as well as regional ones, we propose that the Dulaankhan granitic pluton was likely generated in the post-collision setting related to the orogenesis of the Mongol-Okhotsk belt that seems to occur prior to Early Jurassic in the northern Mongolian segment.
\end{abstract}

Keywords: A-type granite, Mongolia-Transbaikalian belt, U-Pb dating

\section{INTRODUCTION}

Since "A-type granite" was first recognized about 40 years ago (Loiselle and Wones, 1979; Collins et al., 1982), a spectrum of alkali-rich granitic rocks has been included in this type, from syenogranite through peralkaline granite, rapakivi granite and charnockite, to fluorine-rich topaz granite (Collins et al., 1982; Whalen et al., 1987; Eby, 1992; Patiño Douce, 1997; Wu et al., 2002; Bonin, 2007). Such a wide range of composition is likely to be one of the important factors that lead to the debate regarding the origin of A-type granites (e.g., Whalen et al., 1987; Bonin et al., 2004; Whalen, 2005; Bonin, 2007). Consequently, a unified model for the generation of A-type granitoids seems not to have reached.

It is widely accepted that A-type granites are

(C) The Author(s). 2019 Open access This article is distributed under the terms of the Creative Commons Attribution 4.0 International License (https://creativecommons.org/licenses/by/4.0/), which permits unrestricted use, distribution, and reproduction in any medium, provided you give appropriate credit to the original author(s) and source, provide a link to the Creative Commons license, and indicate if changes were made. 


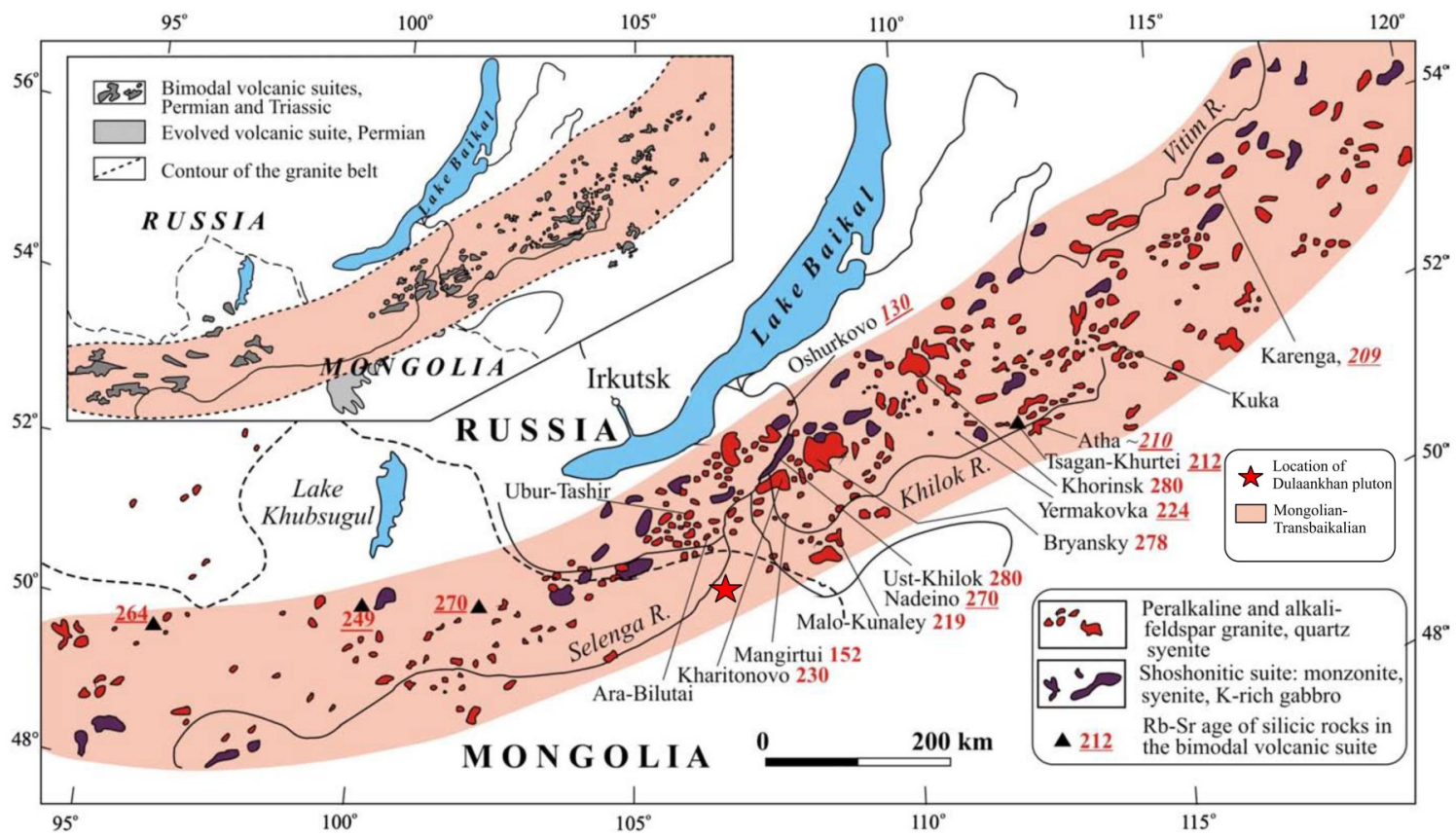

Fig. 1. Distribution of the Late Paleozoic and Mesozoic peralkaline and alkali feldspar granitoids in the Mongolian-Transbaikakalian Belt (Jahn et al., 2009)

produced in extensional settings although the geodynamic mechanisms causing the extensions are not unique, including within-plate extension, rifting extension, post-collision extension, and back-arc extension, etc. Alkaline granitoids are widespread in the Central Asian Orogenic Belt (CAOB) (Jahn et al., 2009), especially within the Mongolia-Transbaikalian belt (MTB; Fig. 1), which contains abundant peralkaline and alkali feldspar granitoids (Fig. 1). However, the age, origin, and tectonic setting of these alkaline granitoids, particularly those in the Mongolian section, are poorly constrained, which hampers understanding of crustal and tectonic evolution of the region. The purpose of this study is to determine the emplacement age(s), to discuss the origin of the Dulaankhan granitic pluton, a typical composite pluton in the MongolTransbaikalian magmatic belt (Fig. 1), and to constrain the tectonic and crustal evolution of the northern Mongolia.

\section{GEOLOGICAL SETTING}

The Dulaankhan area is located in the northern Mongolia, belongs to the MTB, also known as the Mongolian-Transbaikalian rift Province (Zanvilevich et al., 1985). The MTB extends in a NE-SE direction over a distance of $>2500 \mathrm{~km}$ from northern Mongolia to Russia, and varies in width from 150 to $250 \mathrm{~km}$ (Fig. 1). The main part of the MTB is situated in Russia, and the northern Mongolia constitutes its southwestern extension. The MTB has been mapped at scale of 1:50,000 since 1950's (Zanvilevich et al., 1985) and more than 350 alkali-feldspar plutons have been delineated within it so far (Fig. 1; Jahn et al., 2009). Compared with the alkaline plutons in the Russian part, those in the Mongolian segment are relatively less voluminous. Of these, the Dulaankhan pluton is one of the representatives of the alkaline plutons (Fig. 2).

The intrusions of these alkali-feldspar granitoids are contemporaneous (Jahn et al., 2009). Meanwhile, the order of emplacement these alkaline plutons generally starts from alkali feldspar syenite, followed by granite to peralkaline syenite (nordmarkite), finally to peralkaline granite, and the proportion of quartz in syenites increased from 3-6 vol.\% in the earlier to $10-15$ vol. $\%$ in the latest phases. The structures of the plutonic bodies are depended on their sizes (Jahn et al., 2009).

In the MTB, the alkali feldspar granites are normally considered as A-type granite and syenites are closely associated with syenite, both 


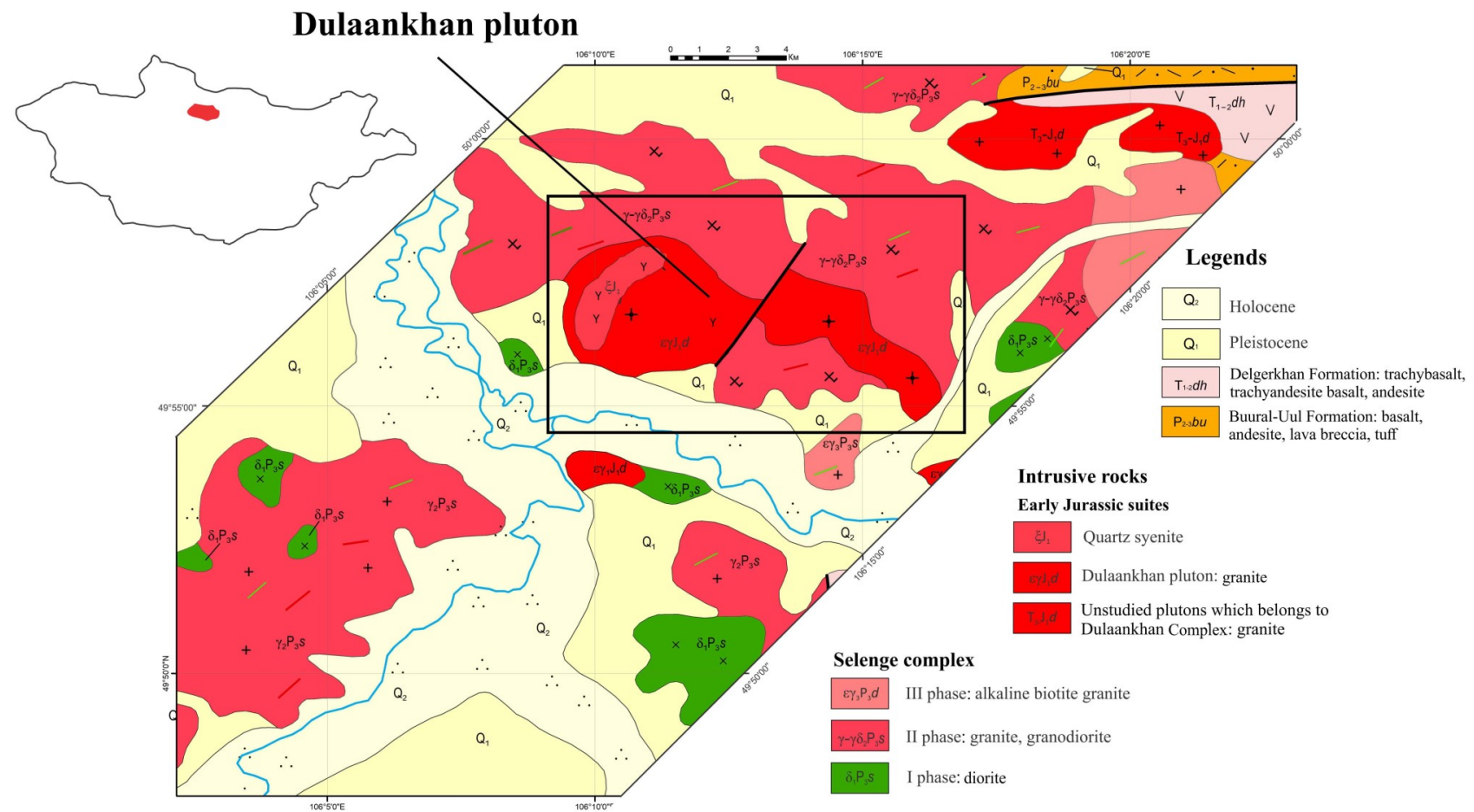

Fig. 2. Geological map of Dulaankhan pluton at 1:100000 scale (Gendenjamts, 2018)

of which were commonly emplaced at the early stages of the granitoid magmatism. Geochemical and $\mathrm{Sr}-\mathrm{Nd}$ isotopic data demonstrated that A-type granite and syenite are cogenetic (Litvinovsky et al., 2015). This means that the syenite and granite magmas were produced from the same source, which insists of juvenile crustal material (Litvinovsky et al., 2015).

The Dulaankhan pluton occurs in the northern Mongolia, about $310 \mathrm{~km}$ north of Ulaanbaatar, the capital of Mongolia. This pluton occupies an exposure area of $47.6 \mathrm{~km}^{2}$ and intrudes into the Middle-Upper Permian Buural-Uul Formation containing basalts, andesite basalts, andesites, lava breccias, tuffs, conglomerates, sandstones, and siltstones, and into the Triassic Delgerkhan Formation comprising trachybasalt, basaltic trachyandesite and andesite. Additionally, it also intrudes the granitoid bodies of the Selenge Complex (Permian). Petrographically, the Dulaankhan pluton is a alkaline granite and is intruded by a small body of quartz syenite (Fig. 2). The intrusive relationships of the granite and the quartz syenite were observed in the field.

\section{ANALYTICAL METHODS}

Samples of the Dulaankhan pluton were analyzed for petrography, major and trace element geochemistry, and radiogenic isotopic age dating. For the petrographic study, thinsections were comprehensively examined by using a microscopy at the Institute of Geology, Mongolian Academy of Sciences (IGMAS). For geochemical analysis, whole-rock samples after removal of altered surfaces were crushed and grilled in an agate mill to $\sim 200$ meshes at the IGMAS. The geochemical analyses were carried out at the SGS Mongolia LLC company (invested by Switzerland) and Field Research Center (FRC) of Mongolian University of Science and Technology (MUST) following the standard procedure. The major and some traceelement (As, Ba, Cr, Cu, V, Zn, Ba, Mo, Nb, Sn, $\mathrm{Ta}$ and $\mathrm{W}$ ) concentrations were determined by using the X-Ray Fluorescence analyzer (XRF76V) and other trace elements and rare earth elements (REE, Th, U, Sr, Rb and Hf) by the Inductively-Coupled Plasma Mass Spectrometry (IC90A and IC90M).

For the U-Pb dating, zircon grains were contracted using heavy mineral contracting methods, and were analyzed by using the LA- 
ICPMS at the Guangzhou Institute of Geochemistry, Chinese Academy of Sciences and at the Geological Institute of Siberian Branch of the Russian Academy of Sciences, Ulan-Ude.

\section{RESULTS}

\section{Petrographic characteristics}

In this study, 24 thin-sections from the Dulaankhan pluton, its country rocks and dykes were examined. As mentioned above, the pluton is a composite body, comprising mainly of granites and a minor mount of quartz syenite. Microscopic observations demonstrate that the granite is coarse to medium-grained and shows a hypidiomorphic to equigranular texture. The mineral assemblage is dominated by K-feldspar (65-69\%), quartz $(25-30 \%)$ plagioclase $(3-5 \%)$ and biotite (1-2\%), with minor amount of opaque minerals. The K-feldspar in this granite displays gridiron twinning, indicating that it is microcline (Fig. 3). So, the Dulaankhan granitic rock is named as K-feldspar granite .

The quartz syenite intruding the granite are porphyritic, with K-feldspar phenocrysts (3.2-4.1 $\mathrm{mm})$ in a fine-grained groundmass, which is composed mainly of K-feldspar (60-65\%), plagioclase $(20-25 \%)$, quartz $(10-20 \%)$, biotite and hornblende $(<5 \%)$. Minor amounts of opaque minerals are also observed in this
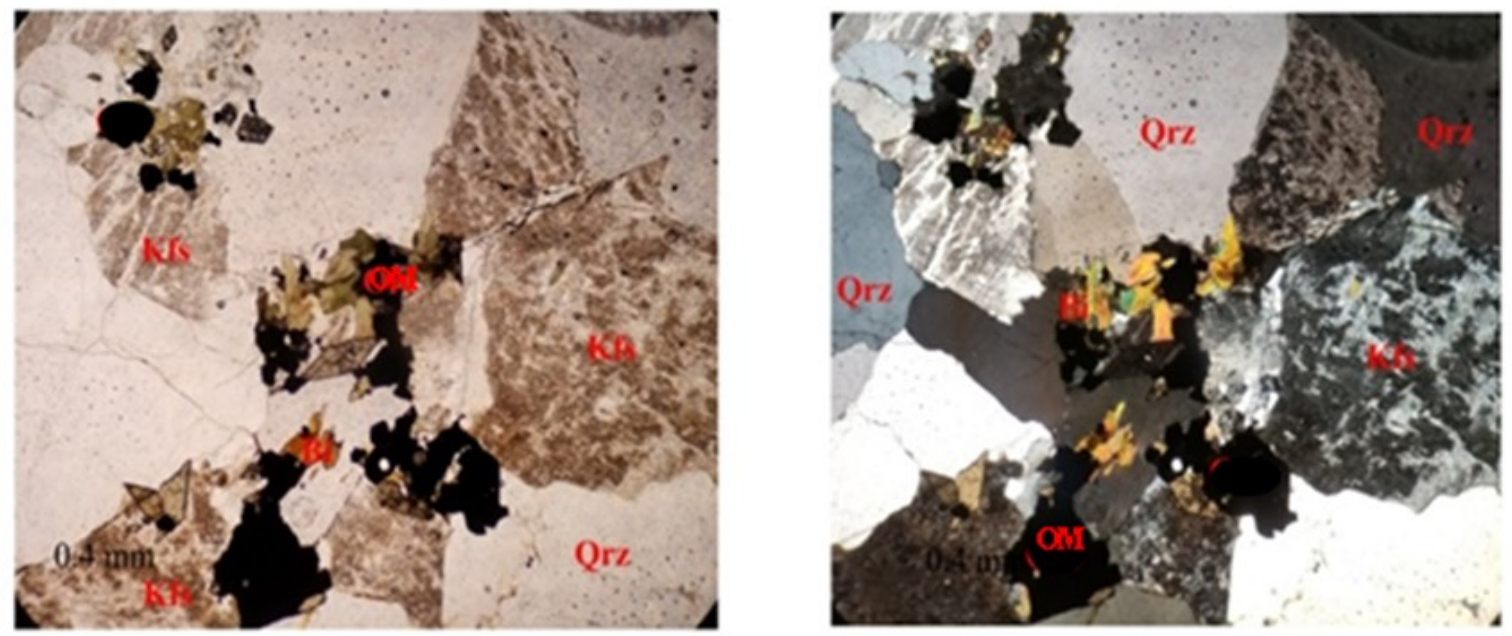

Fig. 3. Microphotographs of the Dulaankhan K-feldspar granite (Dul-02-17) in northern Mongolia (left: plane polarized light picture; right: XPL-cross polarized light image)
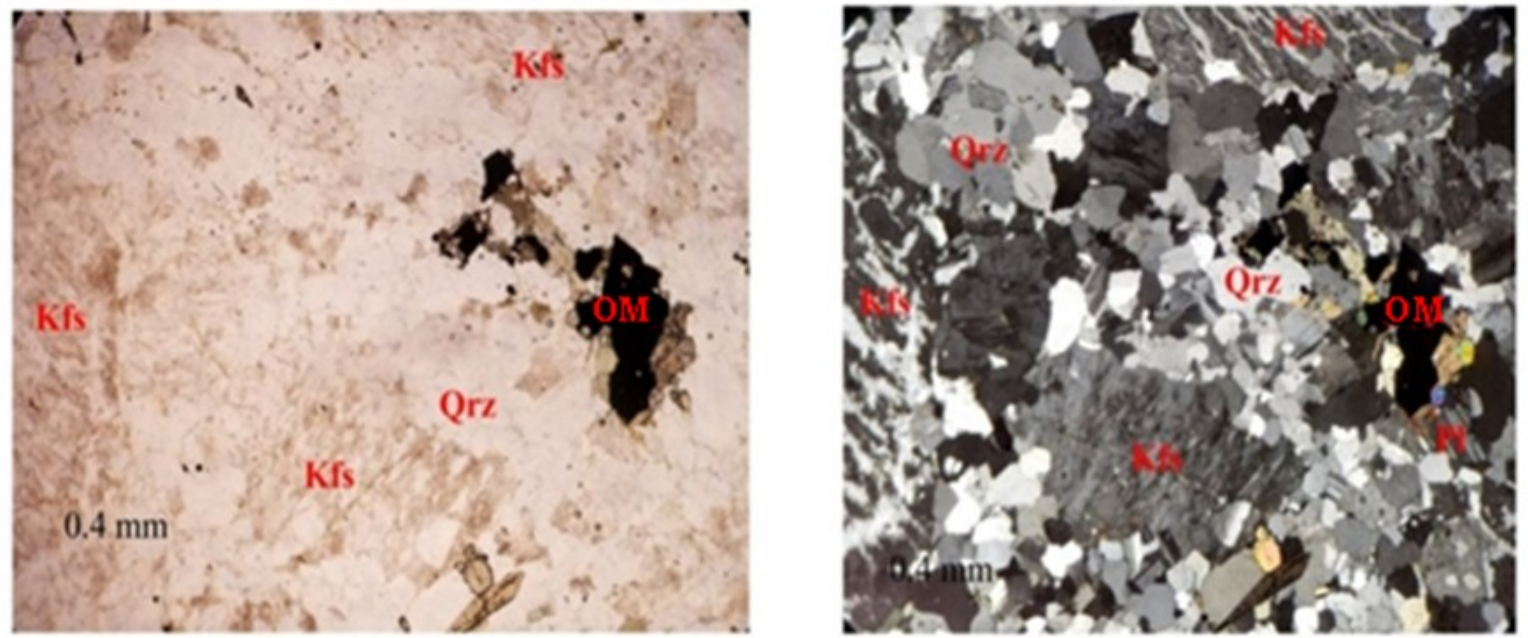

Fig. 4. Microphotographs of a quartz syenite (Dul-01-17) intruding the Dulaankhan granitic pluton in northern Mongolia (left: plane polarized light picture; right: XPL-cross polarized light image) 
syenite. The phenocrystal K-feldspar shows a stria structure, suggesting that it is perthite (Fig. $4)$.

\section{Geochemistry results}

Eight samples were analyzed for major and trace elements, of which 6 samples from the the Dulaankhan pluton and 2 samples from the quartz syenite intruding the granite. The Dulaankhan K-feldspar granite has 77.1-78.13 wt. $\% \mathrm{SiO}_{2}, 12.45-13.79$ wt. $\% \mathrm{Al}_{2} \mathrm{O}_{3}, 0.04-0.2$ wt. $\% \mathrm{MgO}, 0.13-0.34$ wt. $\% \mathrm{CaO}, 3.54-4.89$ wt. $\% \mathrm{Na}_{2} \mathrm{O}$, and 4.44-4.6 wt. $\% \mathrm{~K}_{2} \mathrm{O}$. Compared with the granites, the quartz syenite is relatively low in $\mathrm{SiO}_{2}(\sim 69 \mathrm{wt} \% \%)$ but relatively high in $\mathrm{Al}_{2} \mathrm{O}_{3}$ (15.1-15.36 wt.\%), $\mathrm{Na}_{2} \mathrm{O}$ (5.03-5.19 wt.\%) and $\mathrm{K}_{2} \mathrm{O}$ (5.3-5.7 wt.\%), as well as $\mathrm{CaO}$ (0.45-0.5 wt.\%) (Table 1).

In $\left(\mathrm{Na}_{2} \mathrm{O}+\mathrm{K}_{2} \mathrm{O}\right)-\mathrm{SiO}_{2}$ diagram (Cox, 1979; Wilson, 1989), all the 6 samples of the K-

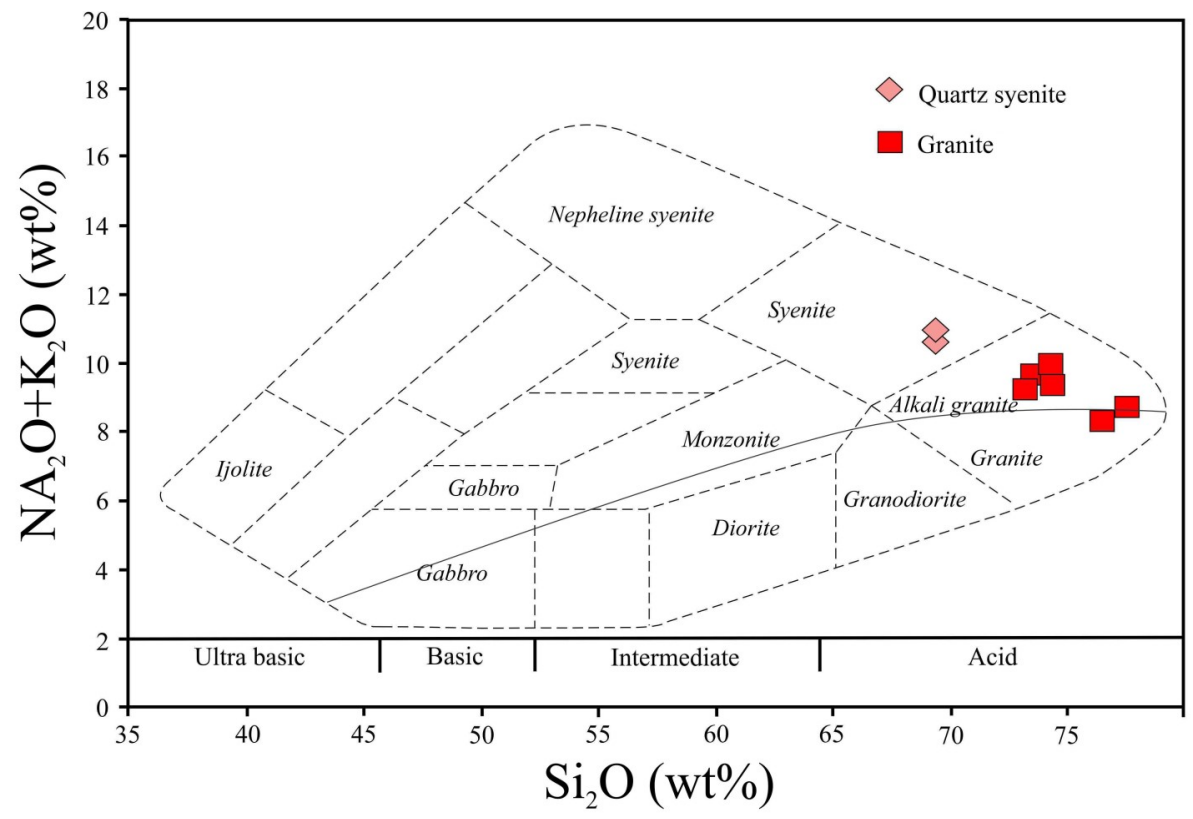

Fig. 5. $\left(\mathrm{Na}_{2} \mathrm{O}+\mathrm{K}_{2} \mathrm{O}\right)-\mathrm{SiO}_{2}$ diagram (Cox, 1979, Wilson, 1989)

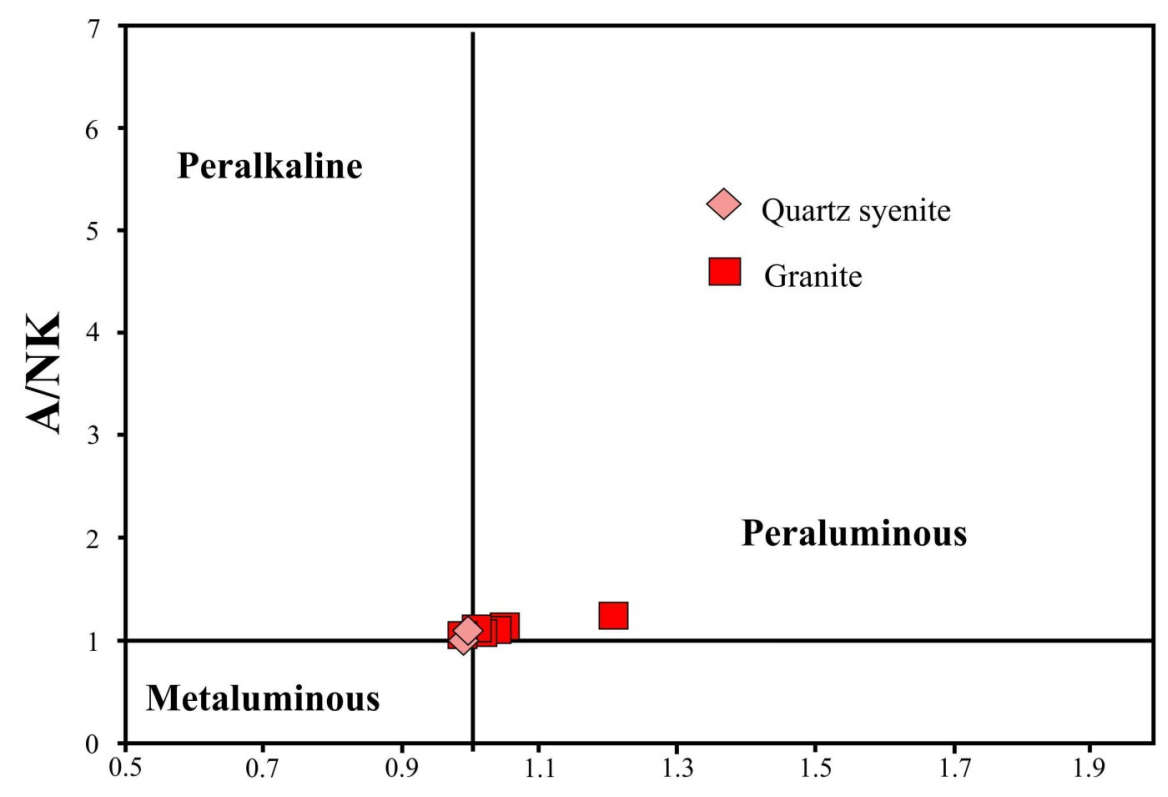

A/CNK

Fig. 6. A/CNK-A/NK diagram (Shand, 1943) 
Table 1. Chemical composition of rocks from the Dulaankhan granitic pluton

\begin{tabular}{|c|c|c|c|c|c|c|c|c|}
\hline Sample no. & Dul-1-17 & Dul-06-17 & Dul-02-17 & Dul-03-17 & Dul-04-17 & Dul-05-17 & Dul-09-17 & G-1 \\
\hline Rock type & \multicolumn{2}{|c|}{ Quartz syenite } & & \multicolumn{5}{|c|}{ Dulaankhan pluton } \\
\hline \multicolumn{9}{|c|}{ Major elements ( \%) } \\
\hline $\mathrm{SiO} 2$ & 69.91 & 69.94 & 74.15 & 74.2 & 74.79 & 77.11 & 78.13 & 74.18 \\
\hline $\mathrm{TiO} 2$ & 0.303 & 0.25 & 0.18 & 0.19 & 0.19 & 0.07 & 0.116 & 0.18 \\
\hline Al2O3 & 15.136 & 15.36 & 13.17 & 13.79 & 13.69 & 13.34 & 12.45 & 13.44 \\
\hline $\mathrm{Fe} 2 \mathrm{O} 3$ & 1.698 & 1.53 & 1.38 & 1.34 & 1.27 & 0.39 & 0.879 & 1.49 \\
\hline MnO & 0.083 & 0.05 & 0.08 & 0.05 & 0.06 & 0.01 & 0.082 & 0.11 \\
\hline $\mathrm{MgO}$ & 0.137 & 0.25 & 0.24 & 0.19 & 0.2 & 0.1 & 0.041 & 0.12 \\
\hline $\mathrm{CaO}$ & 0.455 & 0.5 & 0.34 & 0.29 & 0.31 & 0.13 & 0.305 & 0.23 \\
\hline $\mathrm{Na} 2 \mathrm{O}$ & 5.198 & 5.03 & 4.8 & 4.7 & 4.69 & 3.54 & 4.193 & 4.89 \\
\hline K2O & 5.376 & 5.73 & 4.44 & 4.48 & 4.45 & 4.6 & 4.341 & 4.6 \\
\hline P2O5 & 0.03 & 0.04 & 0.03 & 0.03 & 0.01 & 0.01 & 0.01 & 0.03 \\
\hline LOI & 0.3 & 0.32 & 0.32 & 0.29 & 0.15 & 0.57 & 0.25 & 0.61 \\
\hline Total & 98.329 & 98.98 & 99.08 & 99.52 & 99.76 & 99.8 & 100.554 & 99.98 \\
\hline \multicolumn{9}{|l|}{$\begin{array}{c}\text { Trace elements (in } \\
\text { ppm) }\end{array}$} \\
\hline Al & & 7.62 & 6.67 & & & 5.85 & 6.41 & 5.96 \\
\hline Ba & 57 & 246 & 153 & 139 & & 30 & 50 & 168 \\
\hline $\mathrm{Be}$ & & 5 & 9 & & & 5 & 6 & 11 \\
\hline $\mathrm{Ca}$ & & 0.32 & 0.3 & & & 0.13 & 0.27 & 0.28 \\
\hline $\mathrm{Cr}$ & 89 & 73 & 148 & 64 & & 142 & 113 & 132 \\
\hline $\mathrm{Cu}$ & 0 & 10 & 0 & 0 & & 0 & 1 & 10 \\
\hline $\mathrm{Fe}$ & & 1.25 & 1.17 & & & 0.39 & 0.71 & 0.92 \\
\hline K & & 4.23 & 3.45 & & & 3.24 & 3.54 & 3.41 \\
\hline $\mathbf{L i}$ & & 10 & 40 & & & 32 & 44 & 47 \\
\hline Mg & & 0.09 & 0.08 & & & 0.01 & 0.03 & 0.06 \\
\hline Mn & & 385 & 657 & & & 21 & 629 & 747 \\
\hline $\mathbf{N i}$ & 2 & 5 & 3 & 4 & & 5 & 4 & 5 \\
\hline $\mathbf{P}$ & & 0.02 & 0.01 & & & 0.01 & 0.01 & 0.02 \\
\hline $\mathbf{S c}$ & & 5 & 5 & & & 5 & 5 & 5 \\
\hline $\mathbf{S r}$ & 26 & 80 & 57 & 55 & & 10 & 16 & 44 \\
\hline $\mathbf{T i}$ & & 0.14 & 0.1 & & & 0.04 & 0.07 & 0.08 \\
\hline $\mathbf{v}$ & & 5 & 5 & & & 5 & 5 & 5 \\
\hline $\mathrm{Zn}$ & 46 & 36 & 53 & 45 & & 20 & 32 & 46 \\
\hline Co & 0 & 0.6 & 0.7 & 0 & & 1 & 0 & 1.7 \\
\hline $\mathrm{Ga}$ & 20 & 21 & 21 & 22 & & 25 & 19 & 20 \\
\hline $\mathrm{Ge}$ & & 2 & 2 & & & 2 & 2 & 2 \\
\hline As & & 5 & 5 & & & 5 & 5 & 5 \\
\hline $\mathbf{R b}$ & 133 & 163 & 250 & 292 & & 229 & 217 & 238 \\
\hline $\mathbf{Y}$ & 32 & 16.1 & 13.7 & 19 & & 39.4 & 9.2 & 14.1 \\
\hline $\mathrm{Zr}$ & 438 & 189 & 144 & 246 & & 84.9 & 78.1 & 332 \\
\hline $\mathrm{Nb}$ & 25 & 17 & 24 & 31 & & 11 & 17 & 24 \\
\hline Mo & 21 & 2 & 10 & 9 & & 2 & 2 & 2 \\
\hline $\mathrm{Cd}$ & & 0.2 & 0.3 & & & 0.2 & 0.2 & 0.2 \\
\hline In & & 0.2 & 0.2 & & & 0.2 & 0.2 & 0.2 \\
\hline Sn & & 2 & 2 & & & 4 & 1 & 3 \\
\hline Sb & & 0.1 & 0.6 & & & 0.2 & 0.2 & 0.2 \\
\hline Cs & & 2.5 & 5.4 & & & 6.2 & 2.6 & 8.4 \\
\hline $\mathrm{La}$ & & 52 & 47.5 & & & 16.8 & 34.4 & 34.4 \\
\hline $\mathrm{Ce}$ & & 74.8 & 54 & & & 39 & 41.7 & 58.1 \\
\hline $\mathrm{Pr}$ & & 8.42 & 5.33 & & & 4.83 & 3.91 & 5.05 \\
\hline Nd & & 30 & 16.7 & & & 20.9 & 12 & 15.2 \\
\hline $\mathrm{Sm}$ & & 5 & 2.8 & & & 7.1 & 1.9 & 2.5 \\
\hline Eu & & 0.58 & 0.29 & & & 0.07 & 0.16 & 0.43 \\
\hline Gd & & 3.08 & 1.84 & & & 6.67 & 1.24 & 2.58 \\
\hline $\mathbf{T b}$ & & 0.42 & 0.26 & & & 1.06 & 0.14 & 0.41 \\
\hline Dy & & 2.85 & 1.89 & & & 6.89 & 1.23 & 1.94 \\
\hline Ho & & 0.53 & 0.4 & & & 1.4 & 0.24 & 0.45 \\
\hline $\mathrm{Er}$ & & 1.78 & 1.34 & & & 4.05 & 1.03 & 1.48 \\
\hline $\mathbf{T m}$ & & 0.25 & 0.19 & & & 0.58 & 0.13 & 0.26 \\
\hline $\mathbf{Y b}$ & & 1.8 & 1.7 & & & 3.7 & 1.2 & 1.8 \\
\hline Lu & & 0.32 & 0.32 & & & 0.56 & 0.21 & 0.33 \\
\hline $\mathbf{H f}$ & & 5 & 4 & & & 2 & 3 & 7 \\
\hline $\mathrm{Ta}$ & & 0.8 & 1 & & & 0.5 & 0.7 & 1.5 \\
\hline w & & 18 & 68 & & & 15 & 18 & 1 \\
\hline $\mathrm{Tl}$ & & 0.7 & 0.9 & & & 1 & 0.9 & 1.2 \\
\hline $\mathrm{Pb}$ & 18 & 24 & 22 & 27 & & 25 & 26 & 22 \\
\hline $\mathbf{B i}$ & & 0.1 & 0.1 & & & 3.4 & 0.1 & 0.1 \\
\hline Th & 22 & 18.3 & 17.9 & 34 & & 15.2 & 15.4 & 18.5 \\
\hline $\mathbf{U}$ & & 1.39 & 2.53 & & & 3.64 & 2.85 & 2.56 \\
\hline
\end{tabular}


feldspar granite plot in the "granite" field, straddling the boundary line between the alkaline and subalkaline series, whereas the 2 samples of the quartz syenite in the "syenite" field, belonging to alkaline series (Fig. 5). However, in terms of the $\mathrm{K}_{2} \mathrm{O}-\mathrm{SiO}_{2}$ relation (Rickwood, 1989), both granitic rocks belongs to high-K and calc-alkaline series (Fig. 6). In the $\mathrm{A} / \mathrm{CNK}-\mathrm{A} / \mathrm{NK}$ diagram and all samples plot in an area transitional from the metaluminous to peraluminous fields (Fig. 7).

Primitive mantle-normalized spider diagram shows that the Dulaankhan pluton is enriched in large ion lithophile elements (LILEs), such as

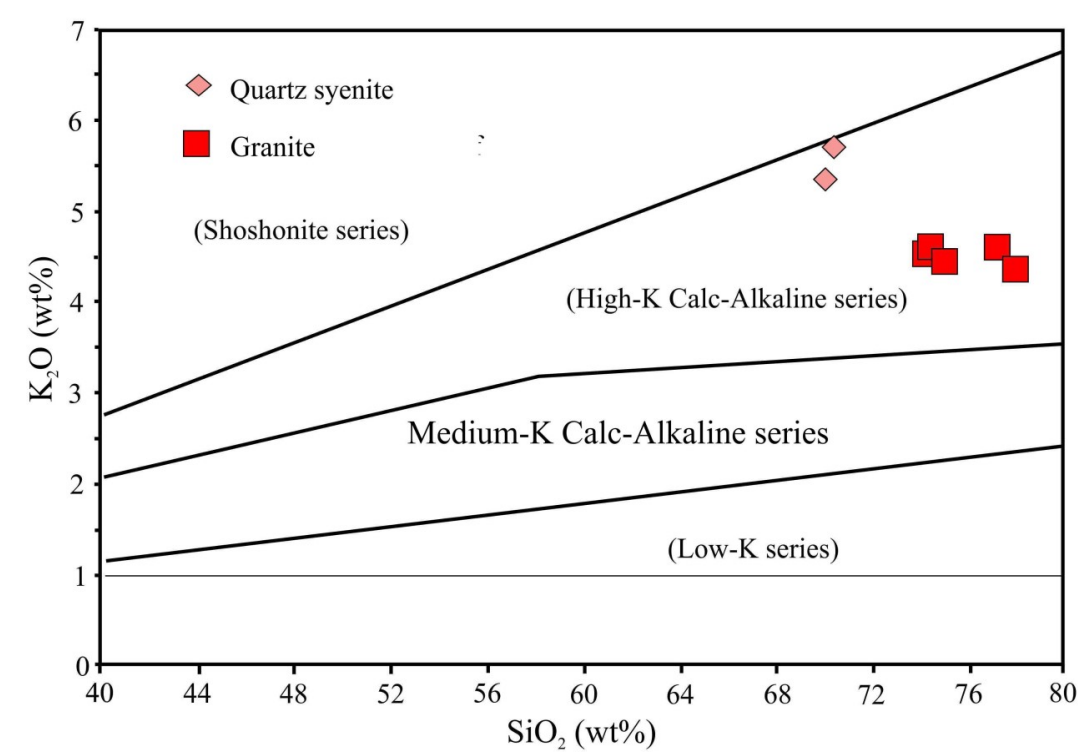

Fig. 7. $\mathrm{K}_{2} \mathrm{O}-\mathrm{Si}_{2} \mathrm{O}$ diagram (Rickwood, 1989)

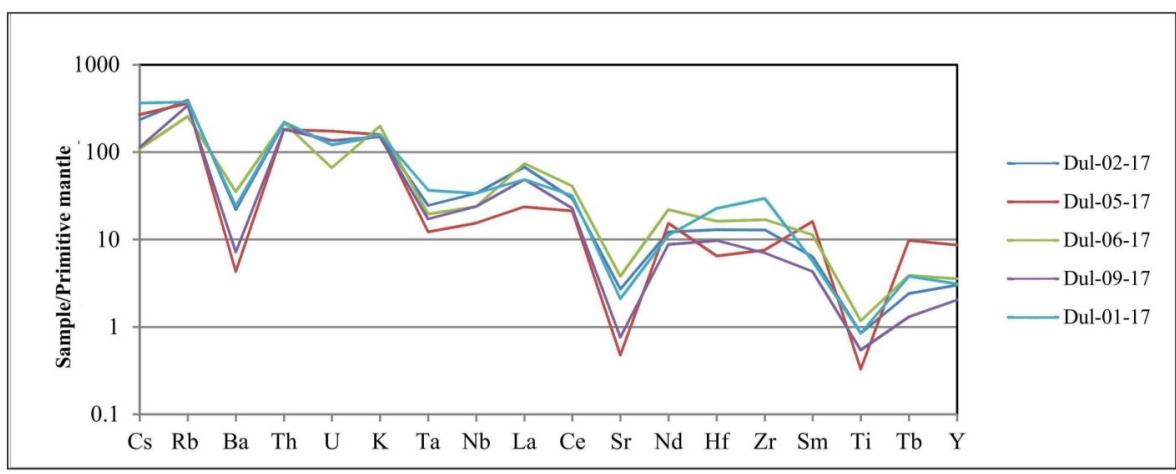

Fig. 8. Primitive-mantle-normalized (Sun and McDonough, 1989) spider diagram

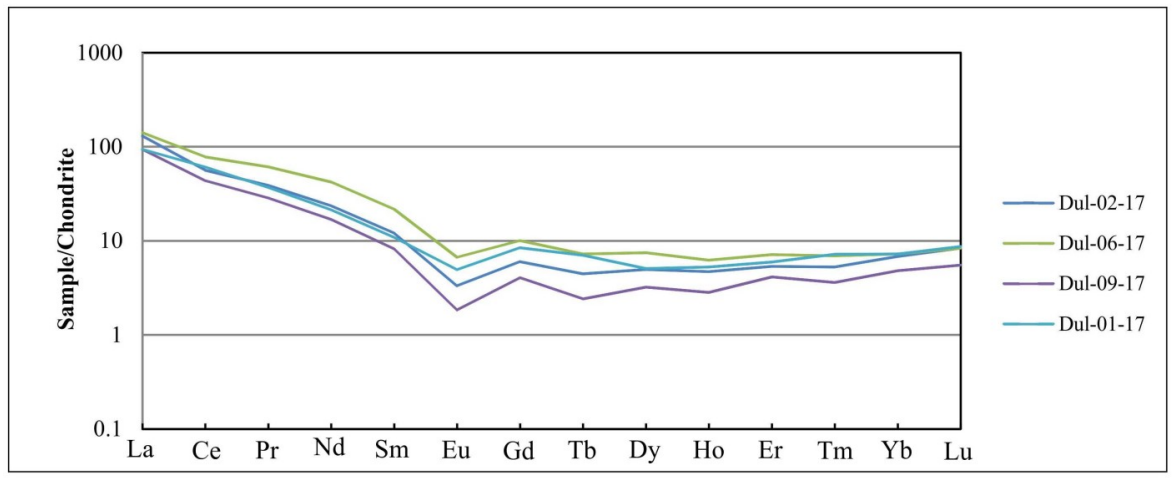

Fig. 9. Chondrite -normalized (Taylor and McLennan, 1985) REE spider diagram 

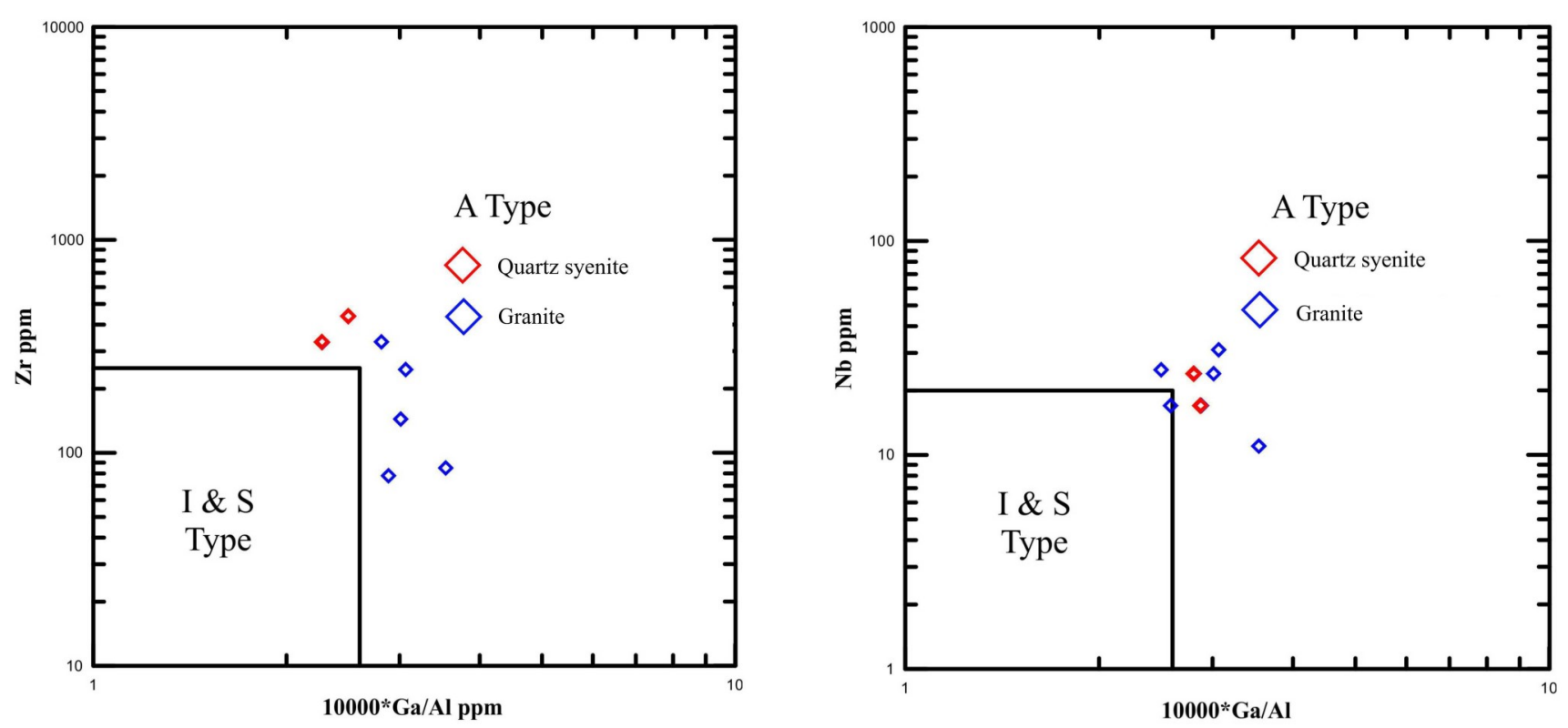

Fig. 10. $10,000 * \mathrm{Ga} / \mathrm{Al}$ vs. $\mathrm{Zr}$ and $\mathrm{Nb}$ diagram (Whalen et al., 1987) showing the A-type granite natures of the Dulaankhan pluton in northern Mongolia

$\mathrm{Rb}, \mathrm{K}$ and $\mathrm{Cs}$ relative to high field strength elements (HFSEs; e.g. $\mathrm{Nb}, \mathrm{Ta}$ and $\mathrm{Ti}$ ). Moreover, negative anomalies of $\mathrm{Sr}$ and $\mathrm{Ba}$ in this diagram are evident, which is consistent with the observation that no or minor amounts of plagioclase exists in the rocks. This pattern of the trace element distribution is similar to that of A-type granites (Fig. 8). The chondritenormalized REE patterns (Taylor and McLennan, 1985) are characterized by enrichment in LREE with respect to HREE, with some extents of negative Eu anomaly (Fig. 9).

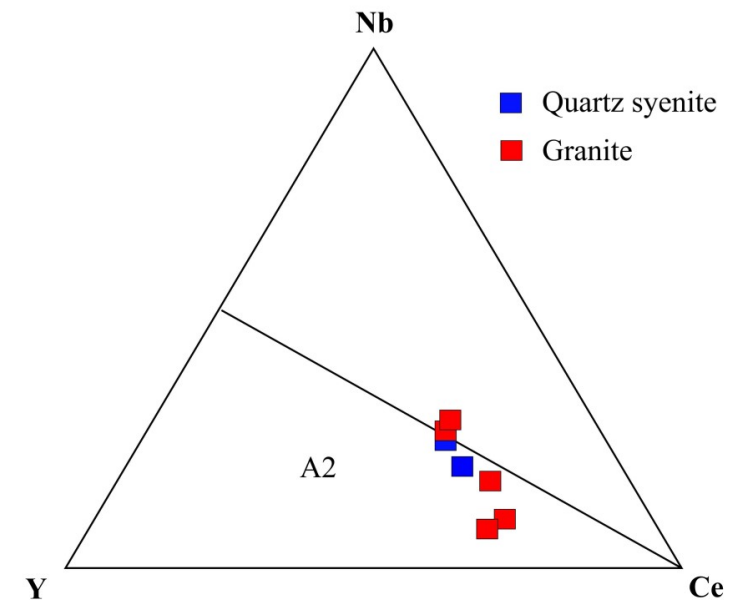

Fig. 11. Y-Nb-Ce diagram showing the A2-type characteristics of the Dulaankhan pluton in northern Mongolia
In the $10,000 * \mathrm{Ga} / \mathrm{Al}$ vs $\mathrm{Zr}$, $\mathrm{Nb}$ diagrams (Whalen et al., 1987), all the Dulaankhan samples and 2 samples of quartz syenite plot into the "A-type granite" field (Fig. 10). The Dulaankhan pluton with samples of quartz syenite show A2-type characteristics in terms of $\mathrm{Y}-\mathrm{Nb}-\mathrm{Ce}$ diagram (Fig. 11).

\section{Geochronology}

Two samples Dul-03-17 (N 4956'30.4", E $106^{\circ}$ 09'53,2") and Dul-06-17 (N 49 57'45,6", E $106^{\circ}$ $\left.10^{\prime} 57,7^{\prime \prime}\right)$, which were respectively collected from the Dulaankhan K-feldspar granite and the quartz syenite intruding the granite, were selected for LA-ICPMS zircon U-Pb dating. Zircon grains from the 2 samples display similar morphological and inner textural features; they are light brownish or colorless, subhedral to prismatic, and generally 50-200 $\mu \mathrm{m}$ long, and show well-developed oscillatory zoning, indicating a magmatic origin for the zircon.

Totally 23 analyses were made on 23 zircon grains of the granite (Dul-03-17). Of these, 21 analyses yield a weighted mean ${ }^{206} \mathrm{~Pb} /{ }^{238} \mathrm{U}$ age of $198 \pm 1 \mathrm{Ma}(\mathrm{MSWD}=0.05)$ (Fig. 12). The remaining 2 analyses were omitted from the age calculation due to their high discordance. The age of $198 \pm 1 \mathrm{Ma}$ is interpreted to be the emplacement age of the granite. 
For the quartz syenite (Dul-06-17), 30 analyses were done on 30 zircon grains, of which 27 analyses give a weighted mean ${ }^{206} \mathrm{~Pb} /{ }^{238} \mathrm{U}$ age of $180 \pm 1$ Ma $(M S W D=0.07)$ (Fig. 13). Similarly, the remaining three analyses were excluded from the calculation due to high discordance. As a consequence, the age of $180 \pm 1 \mathrm{Ma}$ is interpreted as the formation age of the small body of quartz syenite. These new results indicate that the two generations of magmatism of the Dulaankhan composite pluton have a $\sim 10$ Ma difference though both of them occurred in Early Jurassic.

\section{DISCUSSION}

The Dulaankhan pluton is geochemically characterized by high silica and alkalis and belongs to high-K calc-alkaline series (Figs. 5 and 6), which is common in Phanerozoic orogenic belts. On the other hand, the Dulaankhan granite resembles A-type granites in

Table 2. Isotope data of sample (Dul-03-17) from Dulaankhan pluton

\begin{tabular}{|c|c|c|c|c|c|c|c|c|c|c|c|c|c|c|c|c|c|}
\hline Samples & ratio & 1sigma & ratio & 1sigma & ratio & 1sigma & ratio & 1sigma & age & 1sigma & age & 1sigma & age & 1sigma & age & 1sigma & \\
\hline Analysis & $\begin{array}{l}{ }^{207} \mathrm{~Pb} / \\
{ }^{206} \mathrm{~Pb}\end{array}$ & $\begin{array}{l}{ }^{207} \mathrm{~Pb} / \\
{ }^{200} \mathrm{~Pb}\end{array}$ & 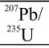 & $\begin{array}{l}{ }^{207} \mathrm{~Pb} / \\
{ }^{233} \mathrm{U} / \\
\end{array}$ & $\begin{array}{l}{ }^{206} \mathrm{~Pb} / \\
{ }^{238} \mathrm{U} / \\
\end{array}$ & $\begin{array}{l}{ }^{206} \mathrm{~Pb} / \\
{ }^{238} \mathrm{U} / \\
\end{array}$ & $\begin{array}{l}{ }^{208} \mathrm{~Pb} / \\
{ }^{232} \mathrm{Th} \\
{ }^{2}{ }^{2}\end{array}$ & 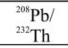 & $\begin{array}{l}{ }^{207} \mathrm{~Pb} / \\
{ }^{206} \mathrm{~Pb} \\
\end{array}$ & $\begin{array}{l}{ }^{2010} \mathrm{~Pb} / \\
{ }^{2006} \mathrm{~Pb}\end{array}$ & $\begin{array}{l}{ }^{206} \mathrm{~Pb} / \\
{ }^{238} \mathrm{U}\end{array}$ & $\begin{array}{l}{ }^{206} \mathrm{~Pb} / \\
{ }^{238} \mathrm{U} / \\
\end{array}$ & $\begin{array}{l}{ }^{2016} \mathrm{~Pb} / \\
{ }^{233} \mathrm{U} /\end{array}$ & $\begin{array}{l}{ }^{207} \mathrm{~Pb} / \\
{ }^{233} \mathrm{U} \\
\end{array}$ & $\begin{array}{l}{ }^{208} \mathrm{~Pb} / \\
{ }^{232} \mathrm{Th} \\
\end{array}$ & $\begin{array}{l}{ }^{203} \mathrm{~Pb} / \\
{ }^{232} \mathrm{Th} \\
\end{array}$ & $\begin{array}{c}\text { Concord } \\
\text { ance }\end{array}$ \\
\hline DOI03-17-1 & 0.053 & 0.0022 & 0.23 & 0.009 & 0.031 & 0.0004 & 0.0100 & 0.0002 & 328.1 & 93.07 & 197.7 & 2.39 & 208.1 & 7.69 & 201.7 & 2.97 & 95.00 \\
\hline DOI03-17-11 & 0.051 & 0.0013 & 0.22 & 0.005 & 0.031 & 0.0003 & 0.0093 & 0.0001 & 224.5 & 56.81 & 198 & 1.84 & 199.9 & 4.31 & 187.8 & 2.4 & 99.05 \\
\hline DOI03-17-12 & 0.050 & 0.0009 & 0.21 & 0.003 & 0.031 & 0.0003 & 0.0095 & 0.0001 & 196.3 & 40.21 & 197.9 & 1.66 & 197.7 & 2.9 & 190 & 1.53 & 100.10 \\
\hline DOI03-17-13 & 0.049 & 0.0008 & 0.21 & 0.003 & 0.031 & 0.0003 & 0.0092 & 0.0001 & 163.7 & 35.09 & 198.2 & 1.61 & 195.5 & 2.43 & 184.9 & 1.4 & 101.38 \\
\hline DOI03-17-15 & 0.049 & 0.0010 & 0.21 & 0.004 & 0.031 & 0.0003 & 0.0094 & 0.0001 & 129.5 & 48.4 & 198.6 & 1.74 & 193.2 & 3.45 & 188.6 & 1.9 & 102.80 \\
\hline DOI03-17-16 & 0.049 & 0.0009 & 0.21 & 0.003 & 0.031 & 0.0003 & 0.0095 & 0.0001 & 163.7 & 40.54 & 197.8 & 1.67 & 195.1 & 2.88 & 190.6 & 1.86 & 101.38 \\
\hline DOI03-17-17 & 0.049 & 0.0008 & 0.21 & 0.003 & 0.031 & 0.0003 & 0.0095 & 0.0001 & 154.7 & 39.52 & 198.5 & 1.67 & 195 & 2.79 & 190.8 & 1.52 & 101.79 \\
\hline DOI03-17-18 & 0.049 & 0.0014 & 0.21 & 0.006 & 0.031 & 0.0003 & 0.0098 & 0.0001 & 170.1 & 63.99 & 198.4 & 1.94 & 196.1 & 4.77 & 197 & 2.24 & 101.17 \\
\hline DOI03-17-19 & 0.055 & 0.0019 & 0.24 & 0.008 & 0.031 & 0.0004 & 0.0095 & 0.0001 & 409.2 & 75.63 & 198.4 & 2.2 & 215.6 & 6.52 & 190.9 & 2.35 & 92.02 \\
\hline DOI03-17-2 & 0.050 & 0.0008 & 0.22 & 0.003 & 0.031 & 0.0003 & 0.0093 & 0.0001 & 194.9 & 38.35 & 198.2 & 1.62 & 197.9 & 2.73 & 186.6 & 1.59 & 100.15 \\
\hline DOI03-17-20 & 0.049 & 0.0013 & 0.21 & 0.006 & 0.031 & 0.0003 & 0.0094 & 0.0001 & 133.8 & 63.51 & 197.7 & 1.92 & 192.7 & 4.63 & 188.2 & 1.84 & 102.59 \\
\hline DOI03-17-21 & 0.050 & 0.0010 & 0.21 & 0.004 & 0.031 & 0.0003 & 0.0094 & 0.0001 & 192.8 & 45.49 & 197.9 & 1.73 & 197.3 & 3.33 & 188.3 & 1.59 & 100.30 \\
\hline DOI03-17-24 & 0.054 & 0.0013 & 0.23 & 0.005 & 0.031 & 0.0003 & 0.0057 & 0.0001 & 383.3 & 51.52 & 197.3 & 1.84 & 212.2 & 4.24 & 114.6 & 1.11 & 92.98 \\
\hline DOI03-17-25 & 0.048 & 0.0010 & 0.21 & 0.004 & 0.031 & 0.0003 & 0.0100 & 0.0001 & 118.3 & 47.52 & 199 & 1.76 & 192.7 & 3.38 & 201.6 & 1.84 & 103.27 \\
\hline DOI03-17-26 & 0.049 & 0.0007 & 0.21 & 0.003 & 0.031 & 0.0003 & 0.0094 & 0.0001 & 127.1 & 33.59 & 198 & 1.64 & 192.4 & 2.28 & 189.2 & 1.46 & 102.91 \\
\hline DOI03-17-28 & 0.043 & 0.0040 & 0.18 & 0.016 & 0.030 & 0.0006 & 0.0093 & 0.0004 & 0.1 & 50.3 & 193.5 & 4.03 & 168.6 & 14.06 & 187.7 & 8.19 & 114.77 \\
\hline DOI03-17-29 & 0.048 & 0.0009 & 0.21 & 0.004 & 0.031 & 0.0003 & 0.0097 & 0.0001 & 118 & 45.07 & 198.6 & 1.74 & 192.3 & 3.18 & 194.8 & 2.12 & 103.28 \\
\hline DOI03-17-3 & 0.050 & 0.0016 & 0.21 & 0.006 & 0.031 & 0.0003 & 0.0090 & 0.0002 & 174.8 & 71.48 & 197.9 & 2 & 196 & 5.36 & 181.3 & 3.4 & 100.97 \\
\hline DOI03-17-30 & 0.051 & 0.0009 & 0.22 & 0.004 & 0.031 & 0.0003 & 0.0094 & 0.0001 & 219.1 & 41.46 & 199.1 & 1.73 & 200.4 & 3.08 & 190 & 1.65 & 99.35 \\
\hline DOI03-17-5 & 0.050 & 0.0007 & 0.22 & 0.003 & 0.031 & 0.0003 & 0.0095 & 0.0001 & 204.2 & 33.75 & 198.1 & 1.58 & 198.5 & 2.36 & 190.6 & 1.49 & 99.80 \\
\hline DPI03-17-6 & 0.050 & 0.0007 & 0.21 & 0.002 & 0.031 & 0.0003 & 0.0095 & 0.0001 & 182 & 30.31 & 198.4 & 1.56 & 197.1 & 2.04 & 191.5 & 1.25 & 100.66 \\
\hline DPI03-17-7 & 0.050 & 0.0009 & 0.21 & 0.003 & 0.031 & 0.0003 & 0.0093 & 0.0001 & 191.5 & 39.97 & 198.3 & 1.65 & 197.6 & 2.87 & 187.7 & 1.52 & 100.35 \\
\hline DPI03-17-8 & 0.049 & 0.0009 & 0.21 & 0.003 & 0.031 & 0.0003 & 0.0096 & 0.0001 & 133.8 & 41.07 & 198.9 & 1.66 & 193.8 & 2.87 & 192.8 & 1.67 & 102.63 \\
\hline
\end{tabular}

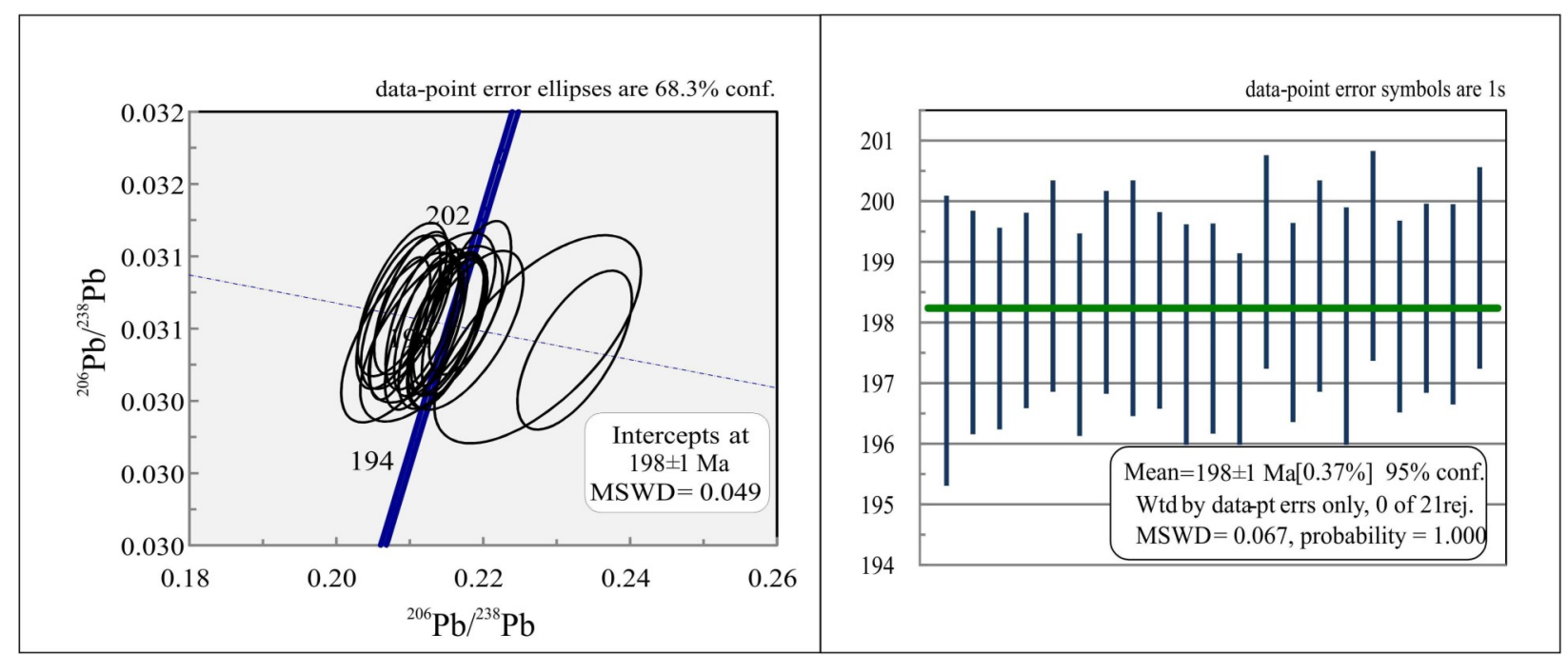

Fig. 12. Concordia and histogram diagrams of the Dulaankhan pluton (sample Dul-03-17) 
Table 3. Isotope data of sample (Dul-06-17) from Dulaankhan

\begin{tabular}{|c|c|c|c|c|c|c|c|}
\hline Samples & \multicolumn{2}{|c|}{ Age } & \multicolumn{4}{|c|}{ Signals, cps } & \multirow[b]{2}{*}{${ }^{238} \mathrm{U}$} \\
\hline Analysis & ${ }^{206} \mathrm{~Pb} /{ }^{238} \mathrm{U}$ & $1 \sigma$ & ${ }^{206} \mathrm{~Pb}$ & ${ }^{207} \mathrm{~Pb}$ & ${ }^{208} \mathrm{~Pb}$ & ${ }^{206} \mathrm{Th}$ & \\
\hline PRB5 & 169.0 & 4.5 & 15730 & 939 & 5711 & 299043 & 457895 \\
\hline PRB1 & 316.3 & 16.7 & 1706806 & 842011 & 2779874 & 3258629 & 11963807 \\
\hline PRB10 & 193.8 & 4.7 & 340290 & 16069 & 259012 & 1351556 & 8813267 \\
\hline PRB11 & 174.5 & 4.4 & 43742 & 2199 & 18206 & 982307 & 1258402 \\
\hline PRB12 & 179.9 & 4.5 & 43451 & 2297 & 16406 & 864405 & 1208425 \\
\hline PRB13 & 178.9 & 4.6 & 37306 & 1872 & 13657 & 755078 & 1048092 \\
\hline PRB14 & 174.2 & 5.0 & 9117 & 505 & 3606 & 193737 & 261556 \\
\hline PRB15 & 180.4 & 4.7 & 21978 & 1001 & 7725 & 405543 & 616504 \\
\hline PRB16 & 174.7 & 4.7 & 13981 & 778 & 4882 & 271816 & 400972 \\
\hline PRB17 & 179.8 & 4.6 & 40479 & 2076 & 11095 & 612307 & 1135291 \\
\hline PRB18 & 173.0 & 4.7 & 14824 & 722 & 4293 & 245984 & 433881 \\
\hline PRB19 & 180.5 & 4.7 & 28497 & 1386 & 12110 & 663634 & 799981 \\
\hline PRB2 & 173.2 & 4.4 & 34901 & 1766 & 8531 & 444408 & 1000116 \\
\hline PRB20 & 178.5 & 4.6 & 39931 & 2007 & 12530 & 686687 & 1132004 \\
\hline PRB21 & 231.9 & 5.8 & 142939 & 7579 & 34383 & 1402300 & 3109452 \\
\hline PRB22 & 229.0 & 5.8 & 101348 & 5160 & 21779 & 909710 & 2240276 \\
\hline PRB23 & 172.0 & 4.5 & 29867 & 1662 & 14687 & 803376 & 876510 \\
\hline PRB24 & 170.5 & 4.5 & 21905 & 1239 & 10206 & 544866 & 648531 \\
\hline PRB25 & 173.3 & 4.6 & 28793 & 1525 & 8357 & 438012 & 842770 \\
\hline PRB26 & 176.0 & 4.7 & 23292 & 1239 & 5780 & 322335 & 673044 \\
\hline PRB27 & 174.2 & 4.9 & 13358 & 722 & 4806 & 270838 & 389945 \\
\hline PRB28 & 175.1 & 4.9 & 13575 & 748 & 4455 & 242983 & 393799 \\
\hline PRB29 & 176.0 & 4.6 & 32351 & 1585 & 11686 & 625455 & 941423 \\
\hline PRB3 & 183.5 & 4.4 & 285769 & 15314 & 215811 & 1263106 & 7702172 \\
\hline PRB30 & 175.0 & 4.6 & 30865 & 1542 & 9827 & 548783 & 903042 \\
\hline PRB4 & 183.6 & 4.5 & 69442 & 3628 & 51740 & 2752507 & 1875499 \\
\hline PRB6 & 177.2 & 4.6 & 23786 & 1254 & 9756 & 517554 & 667817 \\
\hline PRB7 & 178.6 & 4.5 & 58575 & 3032 & 34139 & 1921941 & 1634330 \\
\hline PRB8 & 173.2 & 4.3 & 46724 & 2414 & 15821 & 849097 & 1346267 \\
\hline PRB9 & 185.3 & 4.5 & 270551 & 13292 & 193008 & 1020348 & 7308768 \\
\hline
\end{tabular}
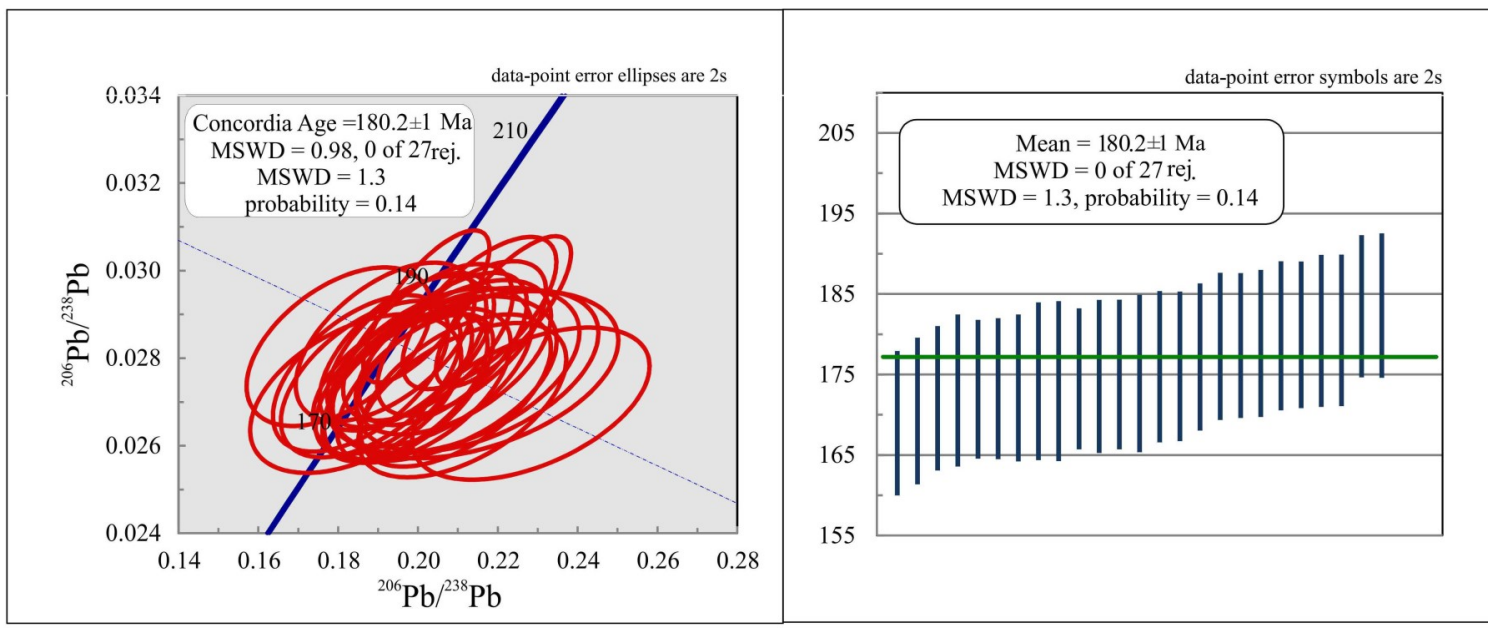

Fig. 13. Concordia and histogram diagrams of the small body of quartz syenite (sample Dul-06-17) 
terms of trace elemental signatures (Fig. 10). These features suggest that the granitic body was produced in an extensional environment.

It is widely accepted that A-type granites can be divided into 2 groups: the A1 group, which is considered to be generated in anorogenic settings and A2 group formed in post-collision/ orogenic settings. The Dulaankhan pluton shows distinct features of A2-type granites (Fig. 12), indicating that this pluton was generated in a post-orogenic setting. Our new age data testify that the Dulaankhan granite was emplaced at the earliest Jurassic at ca. 198 and was in intruded by quartz syenite during Early Jurassic at ca.180 Ma. Considering the regional data, we urge that the post-orogenic environment in which the Dulaankhan A2-type granite generated is related to the orogenesis of the Mongol-Okhotsk belt because this belt is situated to the immediate southeast of the MTB and the orogenesis of the northern Mongolia segment of this belt is confirmed to occur prior to Jurassic.
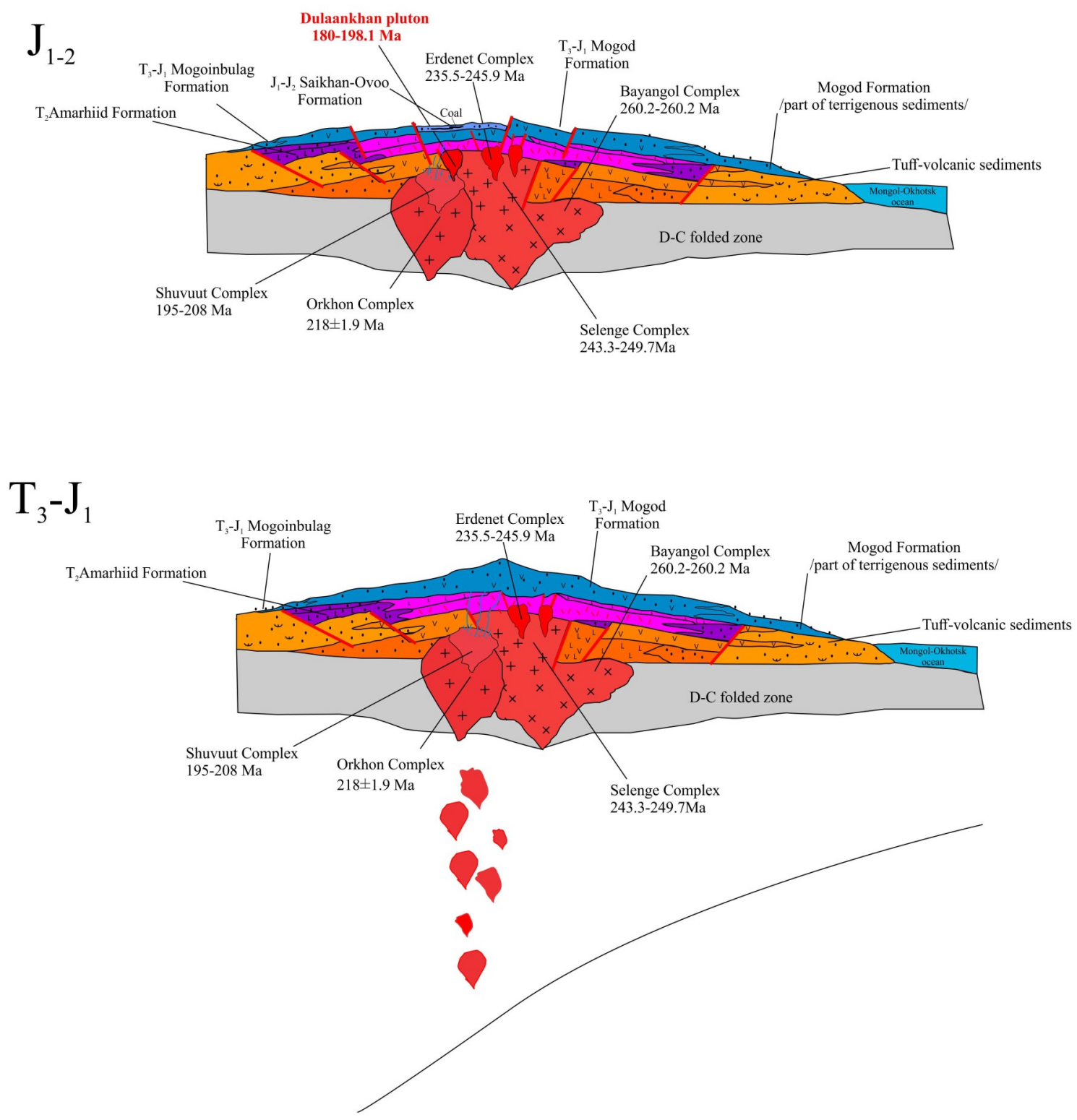

Fig. 14. Cartoon sketches showing the late Triassic (a) and early-middle Jurassic (b) geodynamic setting in which corresponding granitic intrusions formed at the Dulaankhan area in northern Mongolia (based on idea of A.Chimedtseren, unpublished data) 
Regarding the petrogenesis of the Dulaankhan pluton, we believe that it should be generated from partial melting of crustal materials because of its high silica content and its large scale nature in dimension. The magmas have experienced relatively strong plagioclase fractional crystallization, as attested by the lack, or very low contents, of plagioclase in the mineral assemblages of the pluton (Figs. 3 and 4) and the negative anomalies of $\mathrm{Eu}, \mathrm{Sr}$, and $\mathrm{Ba}$ (Figs. 8 and 9). It is noted that the negative anomalies of these elements can also be produced when plagioclase is as a residual phase during partial melting in the source region, meaning that the partial melting likely took place under the condition of plagioclase stability, i.e. a low pressure condition. An extension setting can provide this condition.

On the basis of the data available, we illustrate Paleozoic-Early Mesozoic to Jurassic tectonic and crustal evolution of the Dulaankhan area in northern Mongolia in Fig. 14. From late Triassic to early Jurassic period, the Shuvuut and the Orkhon complexes, which show arc magmatic features, formed probably by the latest northward subduction of the Mongol-Okhotsk oceanic plate (Fig. 14a; Chimedtseren, unpublished data), which led to the closure of the Mongol-Okhotsk Ocean. Following the Mongol-Okhotsk orogenesis, the high-K calcalkaline Dulaankhan A2-type granite formed in the post-collision extensional setting during early-middle Jurassic (Fig. 14b).

\section{CONCLUSIONS}

From current study, following conclusions can be reached:

(1) The Dulaankhan pluton in northern Mongolia is a composite intrusive body, fine to medium grained peralkaline granites and intruded by small body of quartz syenite as well as both two belongs to high-K calc-alkaline series.

(2) Geochemical data shows small body of quartz syenite and peralkaline granites of Dulaankhan magmas were produced singly or together from the same source. But the Dulaankhan pluton formed during Early Jurassic at $198 \pm 1 \mathrm{Ma}$, and quartz syenite is generated at $180 \pm 1 \mathrm{Ma}$, respectively, suggesting that pre- dominantly Jurassic in age.

(3) The Dulaankhan pluton belongs to A2-type granite and was likely generated from partial melting of crustal materials in the post-orogenic/ collision extensional setting related to the orogenesis of the Mongol-Okhotsk belt, which is believed to occur prior to Jurassic in the northern Mongolian segment.

\section{ACKNOWLEDGEMENTS}

The study was supported by "Geodynamic settings and mineralization (W, Sn and REE) of Mesozoic Granitoid Magmatism, Mongolia: Prospective", project (№ SSA 009/2016) of Institute of Geology, MAS. First author is very grateful to colleagues at Institute of Geology, MAS and Professors at the Department of Geology and Hydrogeology, MUST for their continuous support during his MSc study.

\section{REFERENCES}

Bonin, B., Ethien, R., Gerbe, M.C., Cottin, J.Y., Féraud, G., Gagnevin, D., Giret, A., Michon, G., Moine, B. 2004. The Neogene to Recent Rallier-du-Baty nested ring complex, Kerguelen Archipelago (TAAF, Indian Ocean): stratigraphy revisited implications for cauldron subsidence. In: Breitkreuz, C., Petford, N. (Eds.), Physical Geology of HighLevel Magmatic Systems: Geological Society, London, Special Publication, 234, 125-149. $\quad$ https://doi.org/10.1144/ GSL.SP.2004.234.01.08

Bonin, B. 2007. A-type granites and related rocks: Evolution of a concept, problems and prospects: Lithos, 97, 1-29. $\underline{\text { https:// }}$ doi.org/10.1016/j.lithos.2006.12.007

Collins, W.J., Beams, S.D., White, A.J.R., Chappell, B.W. 1982. Nature and Origin of A -Type Granites with Particular Reference to Southeastern Australia: Contributions to Mineralogy and Petrology, 80, 89-200. https://doi.org/10.1007/BF00374895

Cox, K.G., Bell, J.D, Pankhurst, R.J. 1979. The interpretation of igneous rocks. p. 450. https://doi.org/10.1007/978-94-017-3373-1

Eby, G.N. 1992. Chemical subdivision of the Atype granitoids: Petrogenetic and tectonic implications: Geology, 20(7), 641. https:// doi.org/10.1130/00917613(1992) 
020<0641:CSOTAT $>2.3 . \mathrm{CO} ; 2$

Gendenjamts, B. 2018. Geochemistry and geochronology of Dulaankhan massif, Khentii uplift MSc thesis, Mongolian University of Science and Technology, Ulaanbaatar, 60 p. (in Mongolian).

Jahn, B.M., Litvinovsky, B.A., Zanvilevich, A.N., Reichow, M. 2009. Peralkaline granitoid magmatism in the MongolianTransbaikalian Belt: evolution, petrogenesis and tectonic significance: Lithos, 113, 521539.

https://doi.org/10.1016/ j.lithos.2009.06.015

Litvinovsky, B.A., Jahn, B.M., Eyal, M. 2015. Mantle-derived sources of syenites from the A-type igneous suites - New approach to the provenance of alkaline silicic magmas: Lithos, 232, 242-265. https://doi.org/10.1016/ j.lithos.2015.06.008

Loiselle, M.C., Wones, D.R. 1979. Characteristics and origin of anorogenic granites: Abstracts of papers to be presented at the Annual Meetings of the Geological Society of America and Associated Societies, San Diego, California, November 5-8, 11, $468 \mathrm{p}$.

Patino Douce, A.E. 1997. Generation of metaluminous A-type granites by lowpressure melting of calc-alkaline granitoids: Geology, 25, 743-746. https:// doi.org/10.1130/0091-7613(1997) 025<0743:GOMATG $>2.3 . \mathrm{CO} ; 2$

Rickwood, P.C. 1989. Boundary lines within petrologic diagrams which use oxides of major and minor elements: Lithos, 22(4), 247 -263. https://doi.org/10.1016/0024-4937(89) 90028-5

Shand, S.J. 1943. The eruptive rocks: 2nd edition, John Wiley, New York, 444 p.
Sun, S.S., McDonough, W.F. 1989. Chemical and isotopic systematics of oceanic basalts: implications for mantle composition and processes. In: Saunders, A.D., Norry, M.J. (Eds.), Magmatism in Ocean Basin Geological Society of Special Publication, London, 313-345. https://doi.org/10.1144/ GSL.SP.1989.042.01.19

Taylor, S.R., McLennan S.M. 1985. The Continental Crust: Its Composition and Evolution. Blackwell, Oxford.

Whalen, J.B., Curie, K.L., and Chappell, B.M. 1987. A-type granites: geochemical characteristics, discrimination and petrogenesis: Contributions to Mineralogy and Petrology, 95, 407-419. https:// doi.org/10.1007/BF00402202

Whalen, J.B. 2005. A-type granites: N25 years later. Abstracts of the Annual V.M.Goldschmidt Conference, 15th, Moscow, Idaho, May 2005, Geochimica and Cosmochimica Acta 69, A84 Special Supplement.

Wilson, M. 1989. Igneous petrogenesis: A global tectonic approach. Chapman Hall, London, 466 p. https://doi.org/10.1007/978-1 $-4020-6788-4$

Wu, F.Y., Sun, D.Y., Li, H.M., Jahn, B.M., Wilde, S.A. 2002. A-type granites in Northeastern China: age and geochemical constraints on their petrogenesis: Chemical Geology, 187, 143-173. https:// doi.org/10.1016/S0009-2541(02)00018-9

Zanvilevich, A.N., Litvinovsky, B.A., Andreev, G.V. 1985. The Mongol-Transbaikalian alkali -granitoid province. Nauka, Moscow (in Russian). 